\title{
STUDY ON THE MICROSTRUCTURAL EVOLUTION OF DIFFERENT COMPONENT ALLOYS CONSISTING OF B2-NiSc INTERMETALLICS
}

\author{
Zhipeng Yuan - HongBao Cui ${ }^{*}$ - Yatao Chang - XueFeng Guo \\ Materials Science \& Engineering College, Henan Polytechnic University, Jiaozuo 454000, People’s Republic of China
}

\begin{tabular}{|c|}
\hline ARTICLE INFO \\
\hline Article history: \\
\hline Received: 13.9 .2016 \\
\hline Received in revised form: 24.5.2017. \\
\hline Accepted: 18.6 .2017$. \\
\hline Keywords: \\
\hline Intermetallics \\
\hline Microstructural evolution \\
\hline Second phase \\
\hline Rapid solidification \\
\hline$\left(\mathrm{Ni}_{2} \mathrm{Sc}+\mathrm{NiSc}\right)_{\text {eutectic }}$ \\
\hline
\end{tabular}

DOI: http://doi.org/10.30765/er.39.2.5

\section{Introduction}

In recent years, intermetallics have received much attention in material science. Due to their excellent high oxidation resistance, wear resistance, temperature resistance, and other excellent

\begin{abstract}
:
$\mathrm{Ni-50 \% Sc}$ and $\mathrm{Ni-51 \% Sc}$ alloy were prepared with a vacuum arc smelting and water cooled copper mold suction-casting machine. The results showed that the two component alloys consisted of the primary phase B2-NiSc and lamellar $\left(\mathrm{Ni}_{2} \mathrm{Sc}+\mathrm{NiSc}\right)_{\text {eutectic }}$ due to the loss of $\mathrm{Sc}$ during melting. Two groups of alloys underwent $\left(970{ }^{\circ} \mathrm{C}\right.$, $72 \mathrm{~h})$ homogenization heat treatment, and spherical or plate shape $\mathrm{Ni}_{2} \mathrm{Sc}$ particles were dispersed on the B2-NiSc matrix. With the increase of Sc content from $50 \%$ to $51 \%$, the amount of the second phase in the alloy decreases, the microstructure becomes uniform, and the grain gradually changes from long bar to a spherical particle. According to the Jackson boundary theory, the Jackson factor a of B2-NiSc $=0.5<2$, so the interface is rough, which explains that the growth pattern of the B2-NiSc phase is a non-faceted growth. It is consistent with the dendritic growth pattern of the B2-NiSc phase, which is observed from the experiment. After a long heat treatment, the number of vacancies decreases and the microstructure became uniform. The loss rate of $S c$ in rapidly quenched solidification was higher than that after the heat treatment.
\end{abstract}

performance, intermetallics, have been widely applied in various fields, such as the petrochemical, aerospace, and energy power industries [1]. The B2 structure is the body-centered cubic structure. In 2003, Iowa State University and the Ames laboratory Gschneidner K reported that the B2 structure of RM

\footnotetext{
* Corresponding author. Tel.: +86 15893001510;

E-mail address: cuihongbao@hpu.edu.cn
} 
intermetallics (where $\mathrm{R}$ denotes a rare-earth element and $\mathrm{M}$ refers to a transition element or main group element), such as $\mathrm{YAg}, \mathrm{CeAg}, \mathrm{YRh}$ and $\mathrm{DyCu}$ exhibit very good ductility at the room temperature. The intrinsic plasticity of these intermetallics was examined using a tensile test. YAg elongated $27 \%$ before breaking, $\mathrm{DyCu}$ elongated $16 \%$, and $\mathrm{YCu}$ elongated $11 \%$. These values are significantly different from the traditional concept of intrinsic ductility and toughness [2-5]. Further study showed when plastic deformation of RM single crystal begins, that only the $\{100\}<010>$ and $\{110\}<010>$ two independent slip systems were found, which was contrary to the Von-Mises criterion [2-4]. Mulay et al. discovered that the second phase in RM (AgY, CuY, $\mathrm{AgEr}, \mathrm{CuDy}, \mathrm{MgY}$ and $\mathrm{MgCe}$ ) intermetallics had a great influence on the ductility of B2 intermetallics [6-7]. Therefore, revealing the ductility of B2 structure is not only a hot area of research right now, but it also has important theoretical significance. In order to reveal the characteristics of B2's intrinsic plasticity, it is necessary to prepare B2 intermetallics without a second phase. However, it is difficult to achieve B2-NiSc intermetallics without the second phase under the conditions of rapid solidification, due to the loss of Sc during melting [8-10]. Thus, it is quite hard to reveal the intrinsic plasticity of NiSc intermetallics. In fact, no matter how high purity of the starting metal chosen is, the homogeneous singlephase NiSc cannot be obtained.

The key to researching intrinsic plasticity is preparation of a single-phase B2 intermetallics and exploring the action mechanism of the second phase. However, before exploring the second phase's action mechanism, there are several issues that must be understood. First one involves how the second phase is formed. Second, if a second phase exsists, what is the effect of the shape distribution and quantity of this second phase on its organization? Finally, what effect does the second phase have on the B2 intermetallics? It is certain that the formation, distribution, quantity, and nature of the second phase are closely related to the properties of the B2-NiSc intermetallics. In this paper, the characteristics of the B2-NiSc intermetallics were investigated by rapid solidification techniques. The research on the properties of the B2-NiSc intermetallics has laid a foundation for the study of the intrinsic properties of intermetallics, which are of great significance.

\section{The experimental procedure}

The experiment was conducted by smelting under an argon atmosphere and water cooled copper mold suction-casting machine in a vacuum arc. High purity elements of nickel (99.99 wt.\%) and Sc (99.99 wt\%) were alloyed in a vacuum arc furnace to form a NiSc alloy. They were melted four times in order to achieve compositional homogenization. The alloy was poured into a column with a $\Phi 8 \mathrm{~mm}$ diameter in a water-cooled copper mold. The microstructures underwent $\left(970^{\circ} \mathrm{C}, 72 \mathrm{~h}\right)$ homogenization heat treatment.

The samples were then polished and etched with a solution of $1 \mathrm{ml} \mathrm{HF}+4 \mathrm{ml} \mathrm{HNO}_{3}+5 \mathrm{ml} \mathrm{H}_{2} \mathrm{O}$ to examine the microstructure. An optical microscope (MA) and scanning electron microscopy (SEM) were used to examine the microstructure. The chemical composition was determined by energy dispersion Xray spectroscopy (EDS). The transformation temperatures were determined by differential scanning calorimetry (DSC) with a testing temperature scope from $50^{\circ} \mathrm{C}$ to $1400^{\circ} \mathrm{C}$ and a heating-cooling rate of $10^{\circ} \mathrm{C} / \mathrm{min}$. SEM investigations were carried out using a scanning electron microscope operated at $20 \mathrm{KV}$ and linked with an energy dispersive spectrometry (EDS) attachment. The XRD measurements were carried out using an X-ray diffractometer in the range from $20^{\circ}$ to $120^{\circ}$.

The Ni-Sc binary phase diagram shows that the Ni$50 \% \mathrm{Sc}$ alloy was required to obtain single-phase B2NiSc intermetallics. Based on previous experiments, $\mathrm{Sc}$ is easy to lose. Therefore, Ni-50\% Sc and Ni-51\% Sc alloys were prepared for comparison and analysis. Figures 1 and 2 represent the central region 
microstructures of Ni-50\% Sc and Ni-51\% Sc alloy, which were rapidly solidified in a water-cooled copper mold with a $\Phi 8 \mathrm{~mm}$ diameter. The solidification structure is primarily composed of both dendrite and lamellar eutectic structures. The XRD and EDS analyses indicate that the microstructure of the alloy is composed of B2-NiSc and $\mathrm{Ni}_{2} \mathrm{Sc}$. Primary dendrites are present in the B2-NiSc intermetallics, as shown in Fig. 1(a) and Fig. 2(a); the lamellar eutectic structure between the dendrite arm is $\left(\mathrm{Ni}_{2} \mathrm{Sc}+\mathrm{NiSc}\right)_{\text {eutectic}}$, as shown in Fig. 1(b) and Fig. 2(b). Through a comparison and analysis of the solidification structure of $\mathrm{Ni}-50 \% \mathrm{Sc}$ and $\mathrm{Ni}-51 \% \mathrm{Sc}$ alloys, it can be seen that the $\left(\mathrm{Ni}_{2} \mathrm{Sc}+\mathrm{NiSc}\right)_{\text {eutectic }}$ structure reduced with the increase in Sc content from $50 \%$ to $51 \%$. Changes in composition of the Ni$50 \% \mathrm{Sc}$ and $\mathrm{Ni}-51 \% \mathrm{Sc}$ alloys did not change the growth pattern of the primary phase $\mathrm{B} 2-\mathrm{NiSc}$ and $\left(\mathrm{Ni}_{2} \mathrm{Sc}+\mathrm{NiSc}\right)_{\text {eutectic }}$.
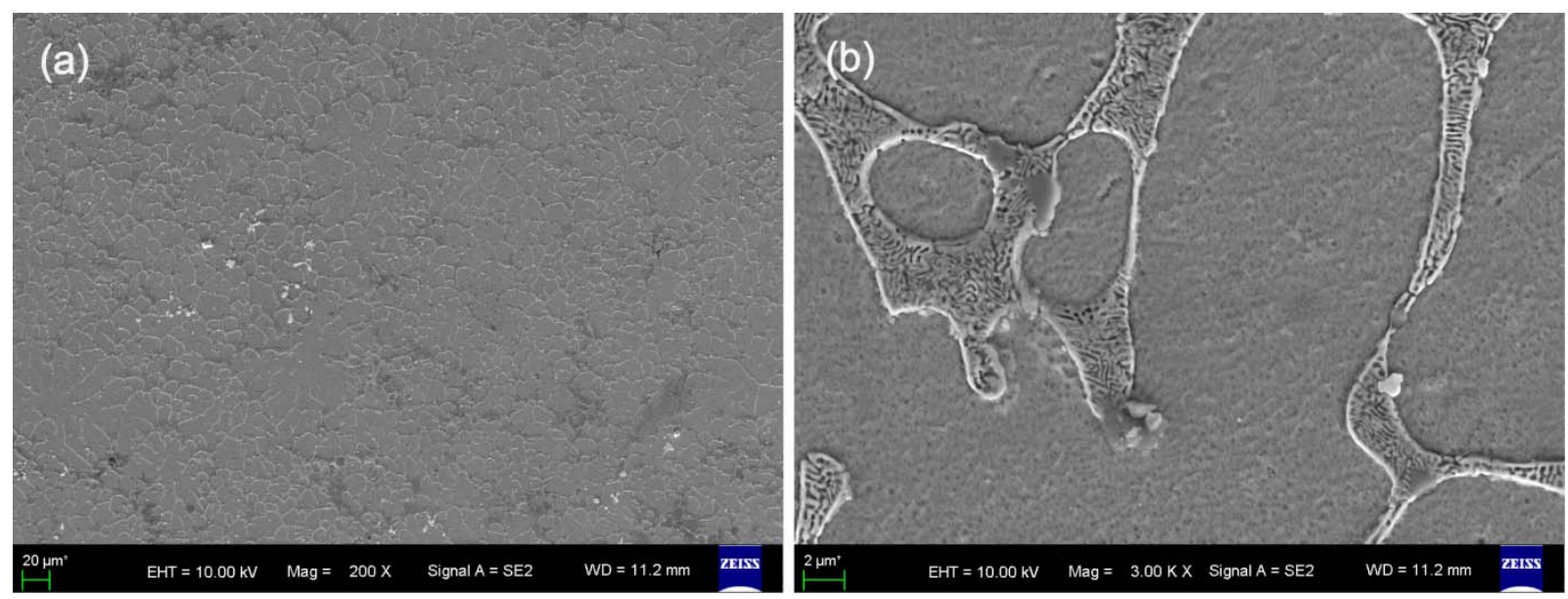

Figure 1. Central region microstructures of rapidly solidified Ni-50\%Sc alloy in a water cooled copper mold with a $\Phi 8 \mathrm{~mm}$ diameter. (a) microstructure; (b) eutectic microstructure between dendrites.
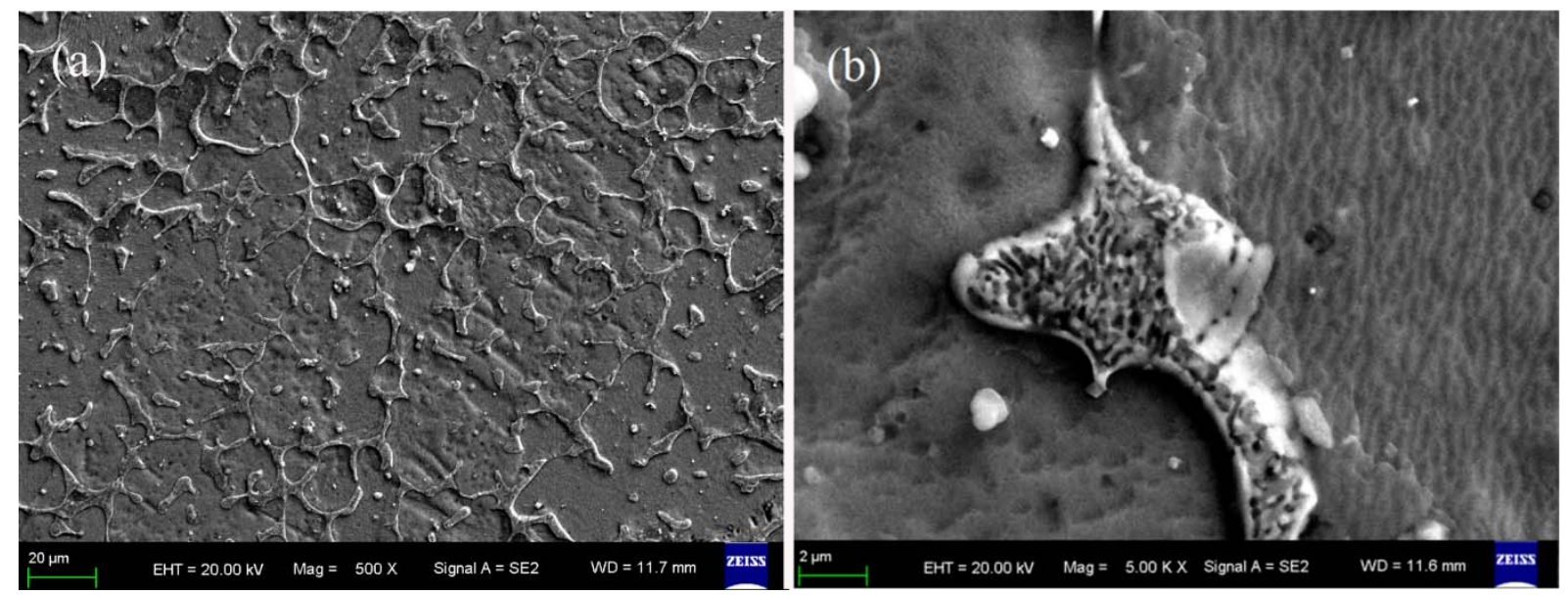

Figure 2. Central region microstructures of rapidly solidified Ni-51\%Sc alloy in a water cooled copper mold with a $\Phi 8 \mathrm{~mm}$ diameter. (a) microstructure; (b) eutectic microstructure between dendrites. 


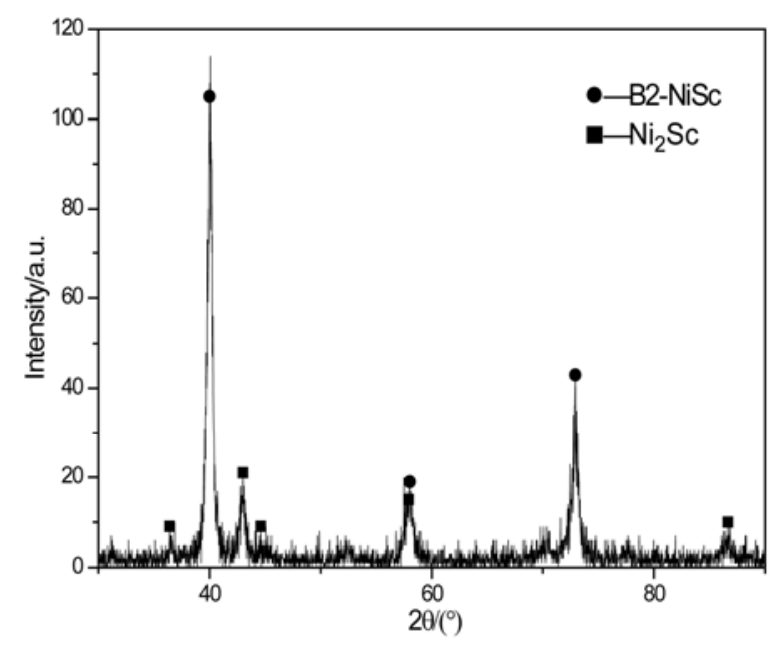

Figure 3. XRD pattern of Ni-50\%Sc alloy by the Centrifugal Casting method with $2.65 \mathrm{~mm}$ thickness.

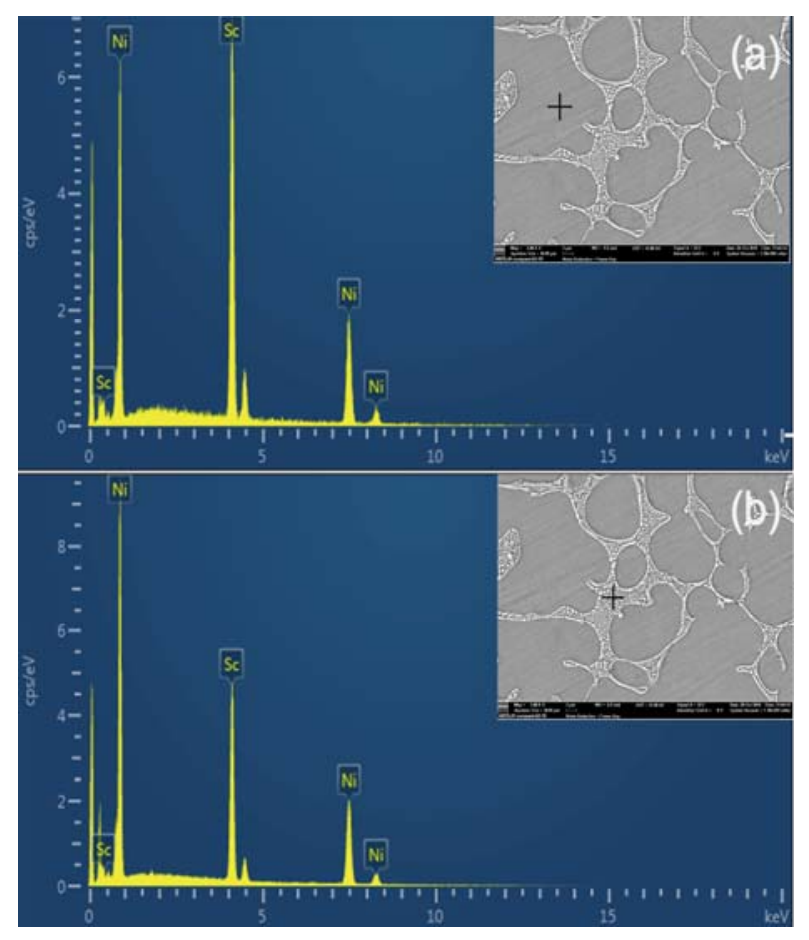

Figure 4. EDS results of Ni-50\%Sc alloy by the Centrifugal Casting method with 2.65 mm thickness: (a) Primary phase; (b) Eutectic microstructure.
In general, the growth pattern of the intermetallics is characteristic of faceted growth. However, as can be seen from Figures 1 and 2, the growth pattern of the B2-NiSc intermetallics is non-faceted. The growth pattern of the primary phase B2-NiSc may be related to the crystal structure of B2-NiSc, but it is more likely to be related to the nature of the solid-liquid interface.

The volume fraction and content of the primary phase B2-NiSc and $\left(\mathrm{Ni}_{2} \mathrm{Sc}+\mathrm{NiSc}\right)_{\text {eutectic }}$ in the $\mathrm{Ni}-50 \% \mathrm{Sc}$ and $\mathrm{Ni}-51 \% \mathrm{Sc}$ alloys were analyzed by Plus ImagePro area calculation software. By applying the lever rule, the proportion of each element in the sample was calculated, and the amount of Sc loss in the experiment was obtained. Based on the phase volumetric analysis of the microstructure with Image-Pro Plus software, the loss of Sc during melting is about $3.20 \% \sim 3.31 \%$.

The rapidly-quenched solidification microstructures of the two component alloys underwent a uniform heat treatment. The central region microstructures of the rapidly solidified alloys after heat treatment are shown in Figure 5. As it can be seen from this figure, after $970^{\circ} \mathrm{C} \times 72 \mathrm{~h}$ of heat treatment, the morphology of the primary phase B2-NiSc dendrite disappeared. The eutectic NiSc in the lamellar eutectic structure together with the primary phase B2-NiSc forms a larger crystal, while $\mathrm{Ni}_{2} \mathrm{Sc}$ in the form of the particles is distributed on the grain boundaries. EDS analysis showed that the matrix was a B2-NiSc phase, while the irregular spherical particles or long Strip particles were the $\mathrm{Ni}_{2} \mathrm{Sc}$ phase. Compared with the two groups in Figure 5, with the increase in Sc content from 50\% to $51 \%$, the number and size of the second phase in the alloy reduced, and the microstructure became more uniform. The $\mathrm{Ni}_{2} \mathrm{Sc}$ phase also shifted from the long Strip particle to the spherical particle. 

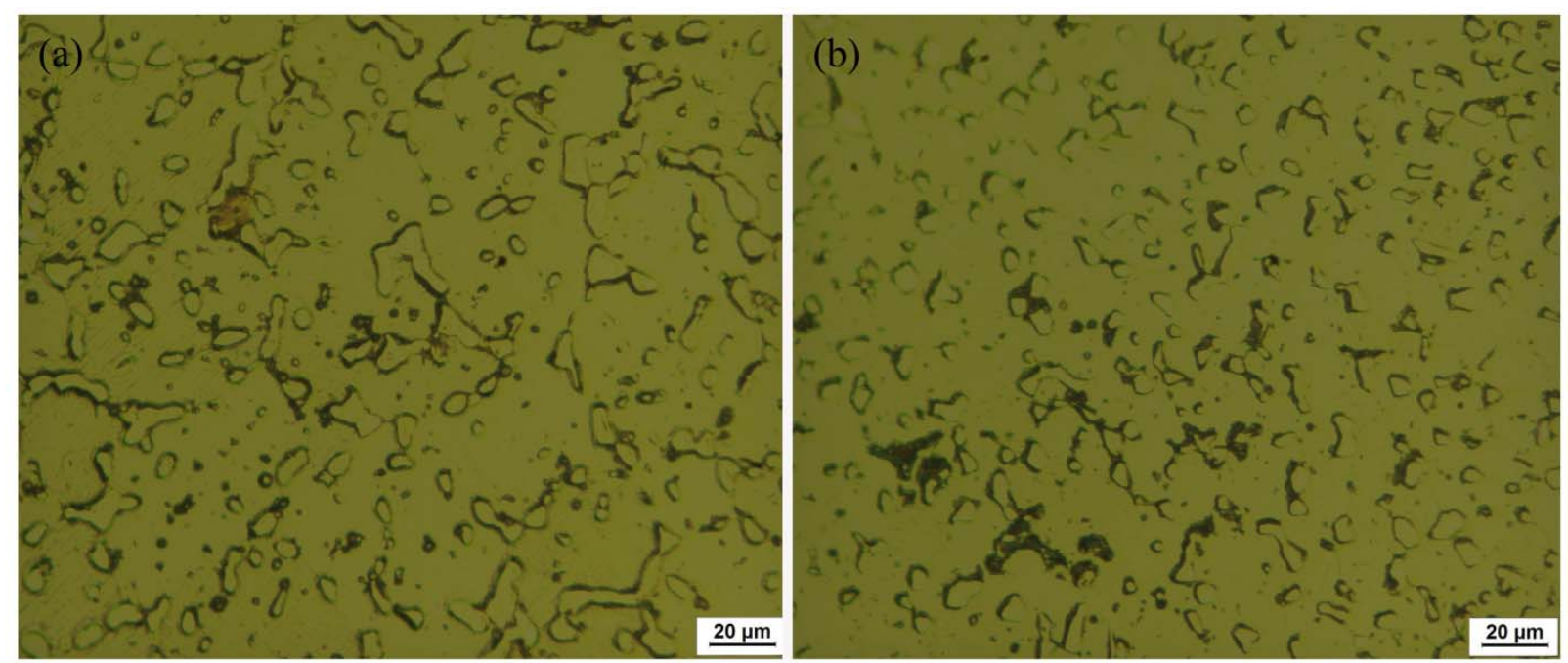

Figure 5. Central region microstructures underwent homogenization heat treatment of rapidly solidified Ni$\mathrm{Sc}$ alloys in water cooled copper molds. (a) Ni-50\%Sc; (b) Ni-51\%Sc.

Similarly, the volume fraction and actual content of the B2-NiSc and the second phase $\mathrm{Ni}_{2} \mathrm{Sc}$ alloy were analyzed with Image-Pro Plus software. Based on the phase volumetric analysis of the microstructure, the loss of Sc during melting is about $2.56 \% \sim 2.92 \%$, which is less than the loss of Sc under the conditions of rapidly-quenched solidification.

\section{The results and discussion}

In general, the growth patterns of the intermetallics is faceted growth [11]. However, the growth pattern of the B2-NiSc intermetallics is non-faceted growth. The growth pattern of the primary phase B2-NiSc may be similar to the B2 type body centered cubic crystal. The solidification structure of this simple crystal structure is more inclined to non-faceted growth. However, according to the Jackson boundary theory, the change of the Gibbs Free Energy in the interfacial layer can be calculated by the approximate calculation of statistical thermodynamics, which can be used to judge the nature of the equilibrium structure of the solid-liquid interface [12]. In accordance with the Jackson boundary theory, the relatively free energy change and the probability of depositing atoms on the interface can be expressed as the following:

$$
\frac{\Delta H_{0}}{\mathrm{~N}_{\mathrm{A}} \mathrm{k}_{B} T_{m}}=\alpha \mathrm{x}(1-x)+x \ln x+(1-x) \ln (1-x),
$$

where $\mathrm{k}_{\mathrm{B}}$ and $\mathrm{N}_{\mathrm{A}}$ are the Boltzmann's constant and the Avogadro's number, respectively; $T_{\mathrm{m}}$ is the melting point of the metal; $\Delta H_{0}$ is the melting enthalpy of an atom; $\eta_{1}$ is the coordination number of atoms in the interface; and $v$ is the coordination number of the crystal. $\alpha$ is an important parameter, which is called the interface phase transition entropy, or Jackson factor [13-14]:

$$
\alpha=\frac{\Delta H_{0}}{\mathrm{k} T_{m}} \cdot \frac{\eta_{1}}{v},
$$

When $\alpha<2$, the interface is rough, typically nonfaceted; otherwise, the interface is smooth, typically faceted.

In order to investigate the phase transition process of the NiSc alloy, the DSC thermal analysis was carried out. The DSC curve of the Ni-50at $\% \mathrm{Sc}$ alloy is illustrated in Fig. 5. In this figure, a point represents heat absorbed by melting of the $\left(\mathrm{Ni}_{2} \mathrm{Sc}+\mathrm{NiSc}\right)_{\text {eutectic}}$; $\mathrm{b}$ point represents heat absorbed by melting of the B2-NiSc dendrite structure. As revealed by the DSC heat flow curves of the NiSc alloy, combined with 
origin software, it can be concluded that the melting enthalpy of the NiSc alloy is $12.83 \mathrm{~kJ} / \mathrm{mol}$. Substituting the value of the melting enthalpy into Eq. (2), the value of $\alpha$ is 0.5 .

According to the Jackson boundary theory, B2-NiSc Jackson Factor $\alpha=0.5<2$, so the interface is rough and non-faceted. It is consistent with the dendrite growth pattern of B2-NiSc phase, which is observed from the experiment. In general, the primary phase B2-NiSc in the dendrite growth form should exhibit good plasticity, which provides the necessary clues for future investigations involving the plastic characteristics.

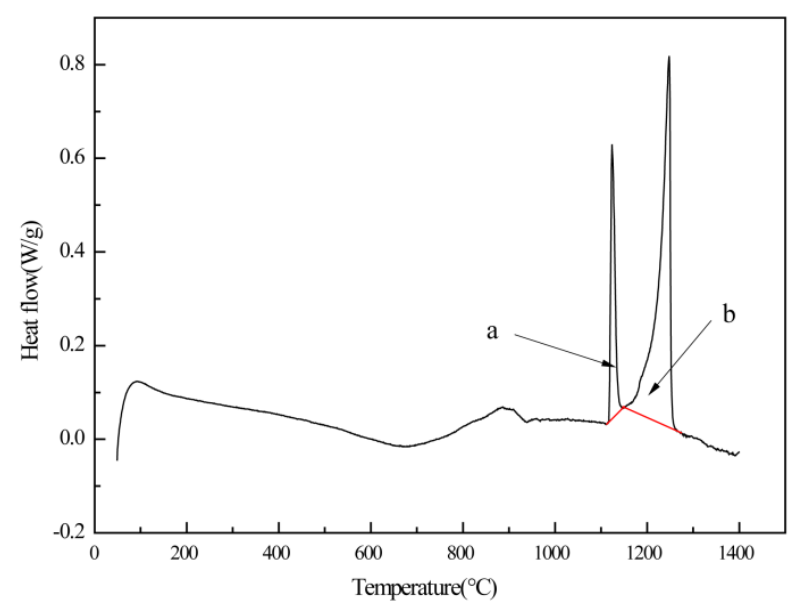

Figure 6. The DSC curve of Ni-50at\%Sc alloy.

The loss of Sc in the structure is not consistent before and after the heat treatment. The liquid metal is composed of a large number of clusters of atoms. The clusters are a kind of ordered structure, and peripheral clusters are a group of disordered atoms. Layer atomic arrangement is in a short-range order at the macro level. There is a large number of vacancies in the crystal, and the number of vacancies increases with the increase in the temperature of the liquidmetal based on the cavity model of the crystal defect. The Arrhenius relationship between the vacancy concentration and temperature is as follows [13]:

$$
n_{\mathrm{v}}=n \exp \left(\frac{-Q_{v}}{R T}\right)
$$

where $\mathrm{k}_{\mathrm{B}}$ and $\mathrm{N}_{\mathrm{A}}$ are the Boltzmann's constant and the Avogadro's number, respectively; $\mathrm{T}$ is Kelvin's temperature; $\mathrm{R}$ is the gas constant; $\mathrm{n}_{v}$ is the number of vacancies in the unit area, $\mathrm{n}$ is the number of atoms in the unit area; and $\mathrm{Q}_{v}$ is the energy required to produce a mole of vacancy. The vacancy formation can be preserved under high temperature by fast cooling through rapid solidification.

In order to clarify the point defect structures of the B2-NiSc intermetallics, the formation enthalpy and formation energy were calculated using the firstprinciples plane-wave pseudo-potential method based on density functional theory [14-15]. The calculated results of the formation enthalpy and formation energy are shown in Figure 7. As it can be seen from this figure, whether single, double, or triple vacancy defects exist, the energy required to generate the $\mathrm{Ni}$ vacancy defect is lower than that required to generate the $\mathrm{Sc}$ vacancy defect. This indicates that the $\mathrm{Ni}$ vacancy defect is easier to generate than $\mathrm{Sc}$ vacancy defect and is also more stable. The number of Ni vacancies is much more than that of Sc that are saved in the rapid solidification process. This causes the relative increase of $\mathrm{Ni}$ content and the relative reduction of Sc content. After a long period of heat treatment, the number of vacancies decreases and the microstructure becomes more uniform. Loss rate of $\mathrm{Sc}$ in the rapid solidification process is higher than that after the heat treatment.

Vacancies exist in the rapidly solidified microstructure and the number of vacancies varies with the change in cooling rate. In this manner, it is theoretically impossible to obtain a complete single phase of the B2-NiSc. There must exist B2-NiSc and a second phase in the structure. The morphology, quantity, distribution, and nature of the second phase have a certain influence on the structure. The mechanism of these factors is the key to the intrinsic plasticity, which provides the direction for follow-up investigations. 


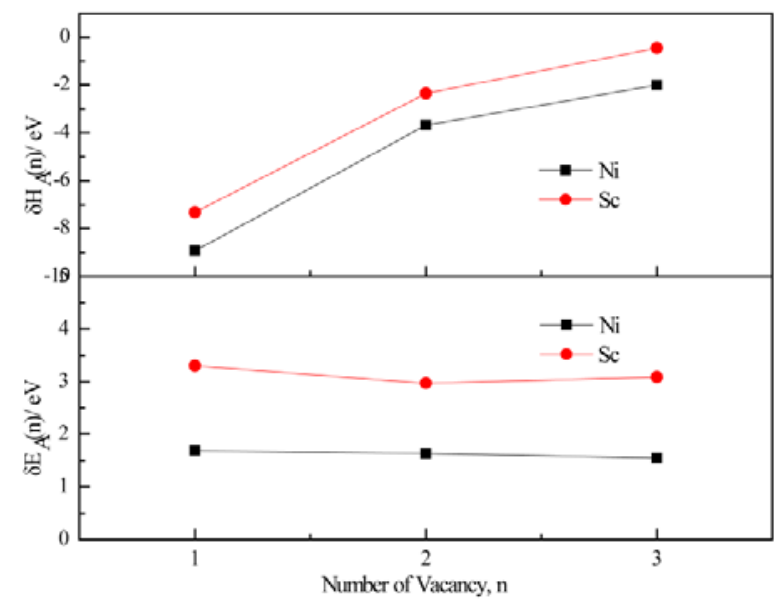

Figure 7. The formation heat per vacancy in $\mathrm{Ni}_{8-n} \mathrm{Sc}_{8}$ or $\mathrm{Ni}_{8} \mathrm{Sc}_{8-n}(n=1,2,3)$ supercells with $n$ vacancies.

\section{Conclusion}

(1) Due to the loss of $\mathrm{Sc}$ in the experimental process, it is theoretically impossible to obtain a complete single phase of B2-NiSc. Two groups of alloys were composed of the primary phase B2-NiSc and lamellar $\left(\mathrm{Ni}_{2} \mathrm{Sc}+\mathrm{NiSc}\right)_{\text {eutectic. }}$

(2) According to the Jackson boundary theory, a Jackson factor $\alpha$ of B2-NiSc $=0.5<2$, so the interface is rough, particularly non-faceted. It is consistent with the dendritic growth pattern of the B2-NiSc phase, which is observed from the experiment.

(3) It can be understood that the number of $\mathrm{Ni}$ vacancies is much higher than that of the $\mathrm{Sc}$ vacancies in the rapid solidification process by the first-principles calculations based on the density functional theory and the cavity model of crystal defects. This causes the relative increase in $\mathrm{Ni}$ content and the relative decrease in Sc content. After a long period of the heat treatment, the number of vacancies decreases and the microstructure becomes uniform. Therefore, the loss rate of Sc in the rapidly quenched solidification process is higher than that after the heat treatment.

\section{Acknowledgements}

This work is supported by the Natural Science Foundation of China (NSFC) under Grant No. 51301063 and No. 51271073 and Talent Introduction Foundation of Henan Polytechnic University (Y2009).

\section{References}

[1] Cao, G.H., Tian, H.N., Liu, N., Li, X., and Ren, Z.M.: Mechanical properties of the B2 $\mathrm{NdAg}$ intermetallic compound, Scripta Materialia, 65(2011), 2, 147-150.

[2] Jr, G.K., Russell, A., Pecharsky, A., Morris, J., Zhang, Z., and Lograsso, T.: A family of ductile intermetallic compounds, Nature Materials, 2(2003), 9, 587-590.

[3] Russell, A.M., Zhang, Z., Gschneidner, K.A., Lograsso, T.A., and Slager, A.J.: Mechanical properties of single crystal $\mathrm{YCu}$ and $\left(T b_{0.88} D y_{0.12}\right) Z n$ B2 intermetallic compounds, Intermetallics, 13(2005), 6, 565-571.

[4] Russell, A.M., Zhang, Z., Lograsso, T.A., Lo, C.C.H., Pecharsky, A.O., and Morris, J.R.: Mechanical properties of single crystal YAg. Acta Materialia, 52(2004), 13, 4033-4040.

[5] Zhang, Z., Russell, A.M., Biner, S.B., Gschneidner, K.A., and Lo, C.C.H.: Fracture toughness of polycrystalline $\mathrm{YCu}, \mathrm{DyCu}$, and YAg, Intermetallics, 13(2005), 5, 559-564.

[6] Stumphy, B., Mudryk, Y., Russell, A., Herman, D., and Gschneidner, K.: Oxidation resistance of B2 rare earth-magnesium intermetallic compounds, Journal of Alloys and Compounds, 460(2008), 1, 363-367.

[7] Mulay, R.P., Wollmershauser, J.A., Heisel, M.A., Bei, H., and Russell, A.M.: X-ray diffraction study of the phase purity, order and texture, of ductile B2 intermetallics, Acta materialia, 58(2010), 7, 2788-2796.

[8] Zhou, S.H., Liu, M., Sun, Z.H., and Chi, Z.Y.: Rapid solidification theory and preparation 
materials, Friend of Science Amateurs, 5(2009), 14, 29-30.

[9] Mei, C.X., Liu, Y., and Zhang, X.A.: Unbalanced effect of rapid solidification, Materials review, 23(2009), 6, 90-93.

[10] Liu, S.J., Huang, and M.Z.: Application of Rapid Solidification Technology, Agriculture network information, 3(2013), 9-10.

[11] Li, Z.Z., Li, S.M., and Fu H.Z.: Directional solidification and growth of Xiaoping's face, Foundry Technology, 31(2010), 9, 1151-1155.

[12] Lv, X.C., He, P., Pan, F., Du, B., Lu, F.J., and Qian, Y.Y.: Effect of $\mathrm{Ag}$ nanopowders on microstructure, hardness and elastic modulus of
Sn-Bi solders, Engineering Review, 34(2014), 2. 63-68.

[13] Askeland, D.R., and Fulay, P.P.: Essentials of materials science and engineering, Cengage Learning, 2013.

[14] Kpesse, G., and Furthmuller, J.: Efficiency of a$b$-initio total energy calculations for metals and semiconductors using a plane-wave basisset, Computational Materials Science, 69(1996), 1, 15-50.

[15] Perdew, J.P., Burker, K., and Ernzerhof, M.: Generalized gradient approximation made simple, Physical Review Letters, 77(1996), 18, 3865-3868. 